Internal Report

DESY M-82-08

March 1982

A THIN LAYER DIELECTRIC NEAR FIELD LASER ACCELERATOR

T. Weiland 
DESY behält sich alle Rechte für den Fall der Schutzrechtserteilung und für die wirtschaftliche Verwertung der in diesem Bericht enthaltenen Informationen vor.

DESY reserves all rights for commercial use of information included in this report, especially in case of filing application for or grant of patents.

"Die Verantwortung für den Inhalt dieses Internen Berichtes liegt ausschließlich beim Verfasser" 
A THIN LAYER DIELECTRIC NEAR FIELD LASER ACCELERATOR

\author{
T. Weiland \\ submitted to the proceedings of the laser acceleration \\ workshop, Los Alamos, Feb. 1982
}

\begin{abstract}
A thin layer dielectric laser accelerator is proposed and analytical expressions are given for accelerating wave structures. All necessary ingredients for a full study of the beam dynamics including longitudinal and transverse collective effects are calculated. Expressions are found for the coupling impedance between the bunched beam and the accelerator as well as for the total parasitic energy loss. Integrals are given for the wake potentials inside the bunch. Dielectric strip line technique may be used to feed the structure with laser light and to focus and steer the light towards the beam.
\end{abstract}

\title{
INTRODUCTION
}

The very intense electromagnetic field produced by a laser beam gave rise to various ideas of how to use the field for acceleration of charged particles to very high energies. We will consider here only the so called "near field accelerators". A material or a grating ${ }^{-9}$ is used to slow down the phase velocity of the laser wave to the particle velocity and to turn the electric field, vector of the transversely polarized wave towards the direction of the beam axis. The partciles travel near to the surface of the material or grating. In specific there exists the idea of using a half space filled with a dielectric medium and laser light coming from the inside towards the surface to the vacuum having a total reflection? This scheme has some practical problems but is the easiest one to describe mathematically. The idea of using gratings ${ }^{2}$ seems to be more realistic but needs further studies of all the collective effects which are very likely to limit strongly the maximum number of particles which can be accelerated in one bunch. A calculation of the beam induced electromagnetic fields on a grating can only be done by means of numerical computations.

In this paper we will describe a dielectric near field accellerator which consists of a thin layer on a meatlic surface. The system has two main components: the feeding in part and the beam line. For the electromagnetic fields on the beam line simple expressions are given. The coupling impedance between the beam and the fields are calculated as well as the fundamental beam loading 10. Integrals describe the beam induced deccelrating and deflecting forces (wake potentials) and the total parasitic energy 1oss. Thus all essential ingredients are available to study the beam dynamics including collective effects such as bunch lengthening or shortening and transverse instabilities and beam break up.

For the feeding in device a mathematical method is described which enables the calculation of coupling coefficients between the 
incoming light wave and the accelerating wave.

A dielectric strip line technique may be used to concentrate the fields in the beam region and to steer the light towards the beam line. Since a dielectric layer and a grating have almost the same wave structure the beam dynamics should also be very similiar in both accelerators.

\section{THE SURFACE WAVES}

The particles to be accelerated travel at the speed $v=\beta c \approx c$ at distance d above the surface of a thin dielectric layer on an infinitely conducting plate of metal, see Fig. 1. In order to

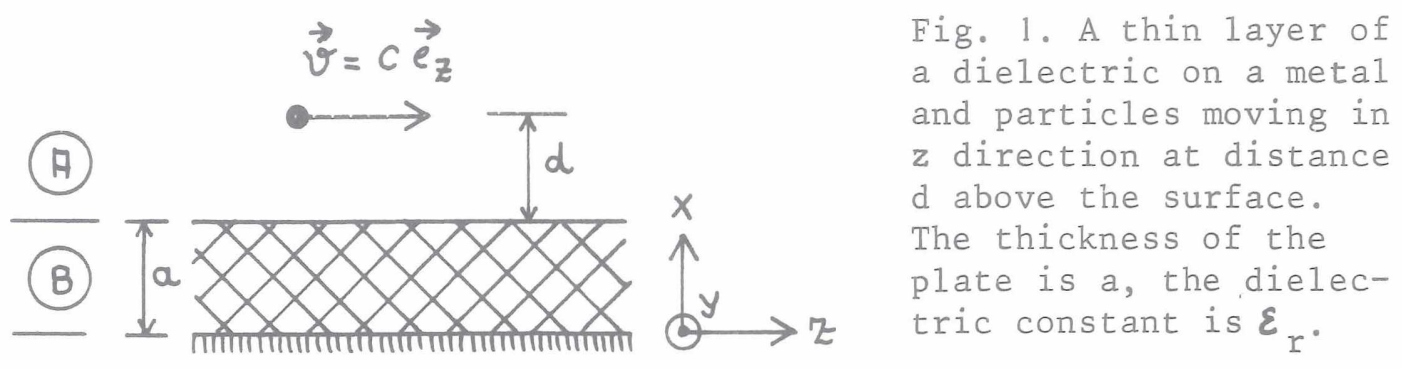

accelerate the partciles efficiently we have to find travelling waves in $+z$ direction with phase velocity equal to $c$. The waves also should carry a finite energy in the fields (evanescent in y direc。). General expressions for such fields may be found in any text book on micro wave theory. For the wave numbers in medium $A$ and $B$ we find : medium A: $k_{0}^{2}=k_{x}^{2}+k_{y}^{2}+k_{z}^{2}, k_{z}=k_{0}, k_{x}^{2}=-K^{2}, k_{y}^{2}=K^{2}$, $k$ real+pos (1) medium $B: \varepsilon_{r} k_{0}^{2}=k_{x}^{2}+k^{2}+k_{0}^{2}, k_{x}=\xi,\left(\varepsilon_{r}-1\right) k_{0}^{2}=K^{2}+\xi^{2}$

The field components may be derived from the two vector potentials:

$$
\begin{aligned}
& \vec{A}_{I}=\vec{e}_{X} E_{I}\left(1 / K Z_{0}\right) \cos \operatorname{Ky} \exp \left(-j k k_{0}(z-c t)\right) \cdot\left\{\begin{array}{l}
\exp (-K(x-a)) ; \quad a \leqslant x \\
\cos \xi x / \cos \xi a ; 0 \leqslant x \leqslant a
\end{array}(3)\right. \\
& \vec{H}_{I}=\operatorname{curI} \vec{A}_{I} \\
& \vec{A}_{I I}=-\vec{e}_{x} E_{I I}(1 / K) \sin \operatorname{Ky} \exp \left(-j k_{0}(z-c t)\right) \cdot\left\{\begin{array}{l}
\exp (-K(x-a)) ; \quad a \leqslant x \\
\sin \xi x / \sin j a ; 0 \leqslant x \leqslant a
\end{array}(4)\right. \\
& \vec{E}_{I I}=\operatorname{curl} \vec{A}_{I I}
\end{aligned}
$$

$E_{I}$ and $E_{I I}$ are the amplitudes of the electric longitudinal field at the surface. Wave type I has no magnetic component in $x$ direction, type II has no electric field perpendicular to the surface. For an arbitrary initial phase of the particles $-\phi=k_{0}(z-c t)$ the electric fields of wave I in the vacuum read as:

$$
\begin{aligned}
\vec{E}_{\text {In }}=E_{\text {In }} \exp \left(-K_{n} d\right) & \left(\vec{e}_{x}\left(\left(k_{n} / k_{0}\right)+\left(k_{0} / K_{n}\right)\right) \sin \phi \cos K_{n} y\right. \\
& +\vec{e}_{y}\left(K_{n} / k_{0}\right) \\
& +\vec{e}_{z}
\end{aligned}
$$


For a given frequency $k_{0}$ the $K_{n}$ have to be solutions of :

$$
\mathrm{K}_{\mathrm{n}} \cdot \varepsilon_{\mathrm{r}}=\xi \tan \xi \mathrm{a} ; \quad\left(\varepsilon_{r}-1\right) \mathrm{k}_{0}^{2^{\mathrm{n}}}=\mathrm{K}_{\mathrm{n}}^{2}+\xi^{2} ; \zeta>0, \mathrm{~K}_{\mathrm{n}}>0
$$

The two curves are illustrated in Fig. 2. The given frequency corresponds to the big circles and it can be seen that only a finite number of solutions (small circles) exist. The cut-off frequencies for the modes are given by poles of the tan-function as:

$$
\mathrm{ak}_{\mathrm{Ic}, \mathrm{n}}=\mathrm{n} \pi / \sqrt{\varepsilon_{\mathrm{r}}-1} ; \mathrm{n}=0,1,2, \ldots
$$

For the second set of waves we find the electric fields above the dielectric at a initial phase $\phi$ to be:

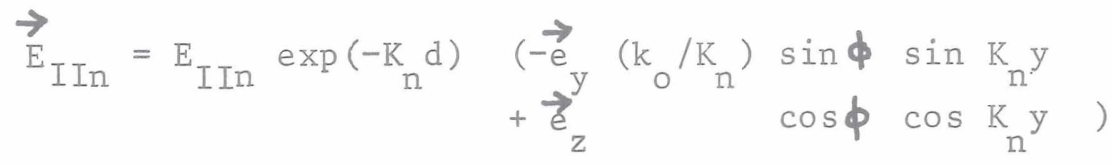

The $\mathrm{K}$ are now solutions of the two equations:

$$
\left.\mathrm{K}_{\mathrm{n}}^{\mathrm{n}}=-\xi \cot \right\} \mathrm{a} ; \quad\left(\varepsilon_{\mathrm{r}}-1\right) \mathrm{k}_{0}^{2}=\mathrm{K}_{\mathrm{n}}^{2}+\xi^{2} ; \zeta>0, \mathrm{~K}_{\mathrm{n}}>0
$$

These equations are illustrated in Fig.3. The cut-off frequencies are found to be:

$$
a_{I I c, n}=((2 n+1) / 2) \pi / \sqrt{\varepsilon_{r}-1} ; n=0,1,2 \ldots
$$

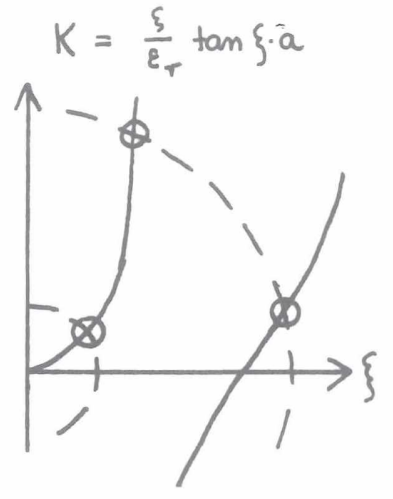

Fig. 2 The two curves giving the eigen solutions $K_{n}$ of waveI

Both types of waves are able to accelerate partciles at the speed of light. The wave numbers $K$ must be real and positiv to keep the energy in the field concentrated in a region near the surface. The y dependence of the field follows with the same wave numbers just by solving the boundary value problem and by asking for waves with phase velocity equal to the speed of light. Since for any given frequency only a finite number of eigen waves are found there must exist other field types on the structure. An arbitrary field cannot be described by a finite set. In order to find such fields we have to give up the condition for the phase velocity. 
Any plane wave falling onto the dielectric surface from the medium A will result in a travelling wave, see Fig. 4. Again there

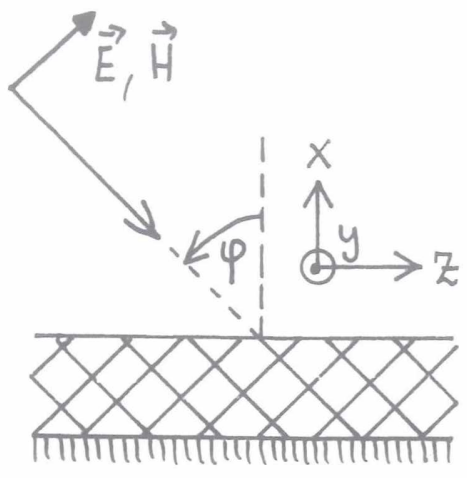

Fig. 4 The two possible polarisations of plane waves falling on the dielectric plate.

exist two types of waves, called III and IV, depending on whether the electric or magnetic field vector is parallel to the $x-z$ plane. Both waves always fulfill the boundary conditions for any incident angle $\varphi$ and at any frequency. There may also be an ang $\overline{l e \psi}$ between the direction of the wave and the $\mathrm{z}$ direction. Since we are interested in waves travelling in $+z$ direction we restrict the angle $\varphi$ to $[0, \pi / 2]$ and assume for any nonzero $\psi$ a pair of waves at $+/-\psi$ with the same amplitude resulting in a sinusoidal dependence in $y$. Without writing down the rather lengthy expressions for the field we conclude that for a given frequency and a given wave number in $y$ the fields are functions of the parameter $\varphi$. An arbitrary field on the dielectric plate may now be written as a sum over the surface waves plus integrals over the plane waves (see for example ref. 11,13):

$$
\vec{E}=\sum_{n=0}^{\infty} a_{I n} \vec{E}_{I n}+\sum_{n=0}^{\infty} a_{I I n} \vec{E}_{I I n}+\int_{0}^{\pi / 2} a_{I I I}(\varphi) \vec{E}_{I I I}(\varphi) d \varphi+\int_{0}^{\pi / 2} a_{I V}(\varphi) \vec{E}_{I V}(\varphi) d \varphi
$$

Each single plane wave of type III or IV has a sinusoidal dependence in $x$ direction and thus carries an infinite energy. The physical constraint is that the total field is of finite energy :

$$
\int_{0}^{\pi / 2}\left(a_{I I I}^{2}(\varphi)+a_{I V}^{2}(\varphi)\right) d \varphi<\infty
$$

Thus the angular distributions a ${ }_{\text {ITI }}$ and $a_{\text {IV }}$ may be written as a sum over orthonormal polynomials which form a complete set on the inter$\operatorname{val}[0, \pi / 2] 12,14$. The scalar product of the polynomials $p_{p}$ and the fields $E_{\text {III }}(\varphi), E_{I V}(\boldsymbol{\varphi})$ yields a set of wave packets with the running index according to $\mathrm{p}_{\mathrm{n}}$ :

$$
\mathrm{e}_{\text {III, IV n }}=\int_{0}^{\pi / 2} \mathrm{P}_{\mathrm{n}}(\varphi) \mathrm{E}_{\text {III, IV } \mathrm{n}}(\varphi) \mathrm{d} \varphi
$$

Any field on the structure may now be written as a sum :

$$
\vec{E}=\sum_{n=0}^{N} \vec{E}_{I n}+\sum_{n=0}^{N_{I}} \vec{E}_{I I n}+\sum_{n=0}^{\infty} \vec{e}_{I I I n}+\sum_{n=0}^{\infty} \vec{e}_{I V n}
$$

The summing index of the two first sums is limited by the number of surface waves at the given frequency. The wave packets have distributions over the phase velocity which extends from c to infinity. These waves never couple to a surface wave if the structure is perfect. These waves will be used to feed the accelerator with light as will be shown later on. 


\section{BEAM DYNAMICS}

The surface waves I and II are the only ones which determine the dynamical behaviour of the particles. For both mode types we find that the accelerating forces fall off exponentially from the dielectric surface as :

$$
\mathrm{F}_{z n I, I I}=e E_{I, I I n} \exp \left(-K_{n} d\right) \cos \phi \cos K_{n} y
$$

The transverse forces due to both waves read as:

$$
\begin{aligned}
& \mathrm{F}_{\mathrm{Xn} I, I I}=e \mathrm{E}_{\mathrm{I}, \mathrm{IIn}} \exp \left(-\mathrm{K}_{\mathrm{n}} \mathrm{d}\right) \sin \phi \cos \mathrm{K}_{\mathrm{n}} \mathrm{y} \\
& \mathrm{F}_{\mathrm{yn} I ; I I}=e \mathrm{E}_{I, I I n} \exp \left(-\mathrm{K}_{\mathrm{n}} \mathrm{d}\right) \sin \phi \sin \mathrm{K}_{\mathrm{n}} \mathrm{y}
\end{aligned}
$$

The force in $\mathrm{x}$ direction tries to push the particles away from the dielectric surface. It has been suggested ${ }^{3}$ to compensate this force by a static magnetic field. Such a field on the dielectric surface could easily produced by a dc current in the metallic plate, Fig. 5.

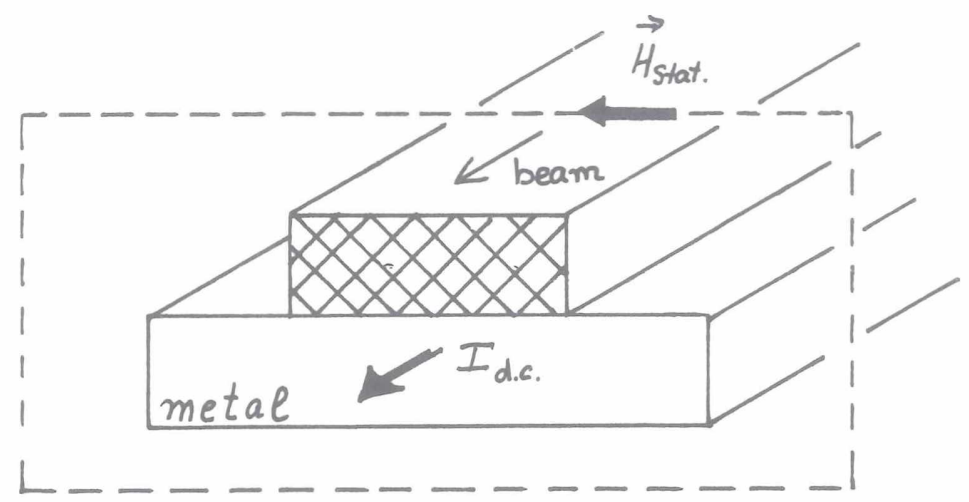

Fig. 5 A magnetostatic field produced by a dc current in the metal plate for compensating the deflecting force in $\mathrm{x}$ direction

The betatron wave numbers are then found to be:

$$
\begin{aligned}
-k_{\beta y}^{2}=k_{\beta x}^{2} & =e E_{I, I I} \cdot K_{n}^{2} \cdot \sin \phi \cdot \exp \left(-K_{n} d\right) /\left(k_{0} \cdot E_{0}\right), \text { with } z_{0}=\sqrt{\mu_{0} / \varepsilon_{0}} \\
H_{y, \text { stat. }} & =E_{I, I I} \cdot K_{n} \cdot \sin \phi \cdot \exp \left(-K_{n} d\right) /\left(k_{0} Z_{0}\right), E_{0}=n o m \cdot \text { energy }
\end{aligned}
$$

The forces in y direction are always defocussing. This fact may be overcome by switching the initial phase of the partciles with respect to the wave from $+\phi$ to $-\phi$ by changing the wave phase as suggested by Kroll and Palmer for grating structures. ${ }^{17}$ In this case the dc field has to alternate also and thus cannot be provided by a dc current in the plate anylonger. Phase switching provides an alternating gradient focussing in the transverse plane but whether a focussing is necessary at all can only be decided for a specific set of parameters. 
The forces induced by the beam itself are proportional to the number of particles per bunch. Since the surface waves I and II are the only ones which can accelerate the bunch these waves are also the only ones which may take energy away from the partciles. The collective effects may be described using the formalism of lossparameters and wake potentials 10 . For the lossparameters per unit length we find:

$$
\begin{aligned}
& k_{n I}^{\prime}=\frac{4 c Z_{0}}{\pi a^{2} M} \exp \left(-K_{n} d\right) \cdot \frac{a^{2} K_{n}^{2}}{\left(1+a^{2} k_{0}^{2} / a^{2} K_{n}^{2}\right) \cdot\left(1+a K_{n} / \cos ^{2} f_{n} a\right)} \\
& k_{n I I}^{\prime}=\frac{4 c Z_{0}}{\pi a^{2} M} \exp \left(-K_{n} d\right) \cdot \frac{a^{2} K_{n}^{2}}{\left(1+a^{2} k_{o}^{2} / a^{2} K_{n}^{2}\right) \cdot\left(1+a K_{n} / \sin ^{2} f_{n} a\right)}
\end{aligned}
$$

$\mathrm{M}$ is the number of half wave lengths in $\mathrm{y}$ direction taken into account for the integration of the total stored energy in the modes. The accelerating voltage is integrated at $\mathrm{y}=0$ and at distance d above the surface. Both lossparameters fall offexponentially from the dielectric surface and both scale like $a^{-2}$. The other terms in the expressions depend not on a but on ak !

The voltage which is induced bỳ a Gaussian bunch into the fundamental accelerating mode may now be calculated by the well known 10 formula $V_{b}=2 q k_{n} \exp \left(-k_{0}^{2} \sigma 2 / 2\right)$ with $q$ as the total charge in the bunch and with $\sigma$ the r.m.s. bunch length. The full formalism of the conventional accelerators may be applied.

The total energy lost by a bunch is somewhat more difficult to evaluate since a dielectric layer is no resonator. The bunch will couple to all frequencies of all the surface modes. Thus we find :

$$
\begin{aligned}
k_{\text {tot }}^{\prime}(\sigma) & =\sum_{n=0}^{\infty} \int_{a k_{I c, n}}^{\infty} k_{I n}\left(a k_{0}\right) \tilde{j}^{\infty}\left(a k_{0}\right) d\left(a k_{0}\right) \\
& +\sum_{n=0}^{\infty} \int_{a k_{I I c, n}}^{\infty} k_{I I n}\left(a k_{0}\right) \tilde{j}_{\left(a k_{0}\right) d\left(a k_{0}\right)}
\end{aligned}
$$

j $(a k)$ is the normalized frequency spectrum of the bunch power spectrum which corresponds to the bunch form factor exp $\left(-k^{2} \sigma^{2}\right)$. The total parasitic losses can now be obtained by subtracting the loss into the accelerating mode from the above expression. The wake forces inside the bunch depend only the the partciles infront of the position $\mathrm{s}$ where the potential is to be calculated. Due to causaltiy a partcile at position s can see fields only from earlier particles. Thus we obtain:

$$
\begin{aligned}
w(s)=2 \cdot q & \sum_{n=0}^{\infty} \int_{a k_{I c, n}}^{\infty} k_{I n}\left(a k_{0}\right) \tilde{j}_{\left(a k_{0}, s\right) \cos (s k) d\left(a k_{0}\right)}^{\infty} \\
+ & \left.\sum_{n=0}^{\infty} \int_{a k_{I I c, n} k_{I I n}\left(a k_{0}\right)}^{\infty} \tilde{j}\left(a k_{0}, s\right) \cos (s k) d\left(a k_{0}\right)\right\}
\end{aligned}
$$


$\widetilde{j}(a k, s)$ is the frequency spectrum of the bunch current cut off at s inside the bunch.

Since the transverse deflecting forces and the longitudinal forces are due to the same waves on the accelerator the full beam dynamics including collective effects may be investigated using the equations given above.

\section{FEEDING THE ACCELERATOR WITH LASER LIGHT}

The wave structure on the dielectric accelerator described above splits into two groups of fields: one which couples to the beam and one which does not couple to the beam having a phase velocity always greater than the speed of light. Unfortunately the waves which do not interact with the beam are the only ones which are able to feed in energy from above the dielectric layer. Thus we need a device which couples the two types of waves. A simple example of

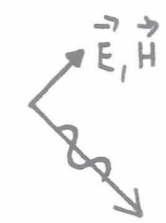

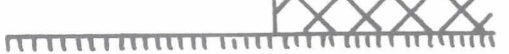

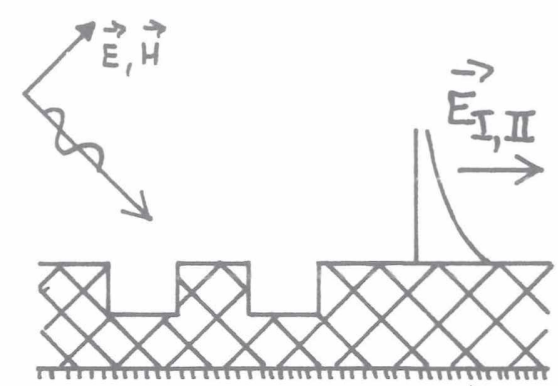

Fig. 6 Two simple structureswhich transfer energy from the incoming laser wave to a surface wave of type I or II

such a structure is given in Fig.6. Both devices are able to transfer energy from an incoming laser wave to surface waves travelling to the right hand side. The frequency may be chosen such that only the surface wave $E_{I, 1}$ is excited. The mathematics to solve this kind of problem is reduced to the problem of Fig.7. By standart eigenmode technique

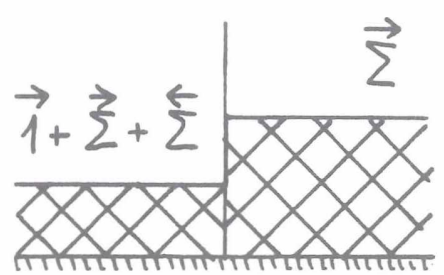

Fig. 7 The basic problem to be solved for calculating the coupling using the incoming field $(\overrightarrow{1})$ plus two sets of forward and backwards travelling waves ( $\hat{\Sigma}, \vec{\Sigma}$ ) on the left hand side the field may be matched to a sum over waves running to the right hand side containing the accelerating mode. After inverting a matrix the transmission coefficient from the incoming laser wave to accelerating wave is known. Since the surface waves must have a y dependence a complementary set of two laser waves is used with the same amplitudes. This fact also solves the problem of keeping the feeding-in dcvice away from the beam axis. The most simple arrangement which could do the job 


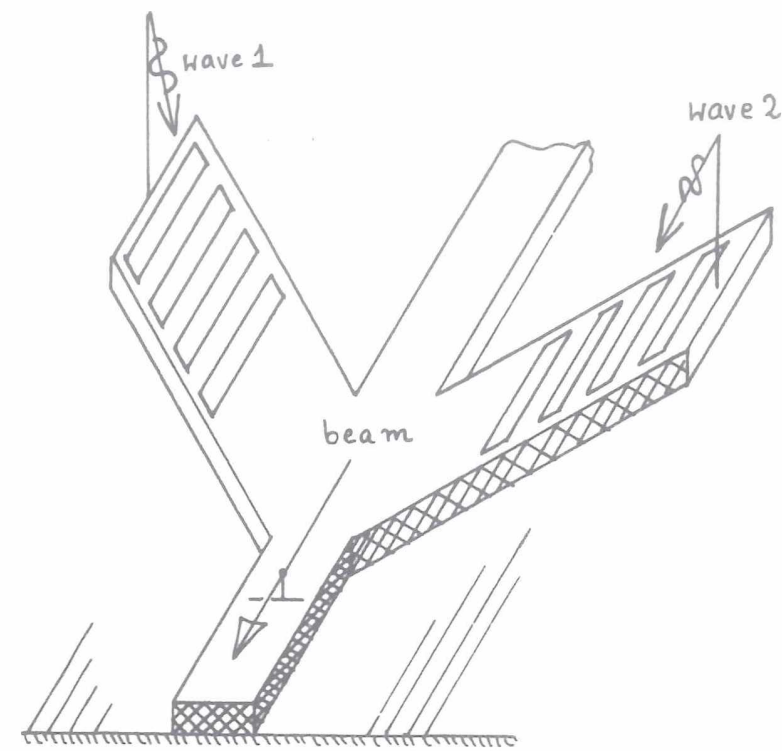

Fig.8 A laser accelerator feeding in the light over two side lines. The strip lines are used to focus and steer the light towards the beam line in the center is shown in Fig. 3. Instead of a dielectric plate a dielectric stripline device is used. The side lines may be adjusted to the light aperture and may be tapered in addition in order to focus the incoming wave towards the beam line. The center strip may be kept small in order to concentrate the fields near the beam. Phase switching can be done by interupting the the dielectric layer under the beam.Although the fields on such strip lines are no longer given by simple expressions they still may be calculated by standard techniques of microwave theory 15,16 .

\section{ACKNOWLEDGEMENTS}

The author wishes to thank A.Chao and H.Henke for helpful discussions.

\section{LITERATURE}

1. Y.Takeda, I.Matsui,Nuc1.Inst.a.Meth.62(1968) 306-310

2. K.Shimoda, App1. Optics 1,33(1962)

3. S.A.Keihfets, 8.Conf.on High Energy Acc., 1971 CERN, 597

4. K.Mizemo,S.Ono,0.Shimoe, Nature, Vo1 253 Jan.1975

5. S.J.Smith,E.M.Purce11, Phys.Rev. 92(1969), 1053

6. R.Rossmanith, Nuc1.Instr,a.Meth.,154(1978),29-39

7. J.D.Lawson, IEEE Trans.on.Nuc1.Science, Vo1NS26, No5, 1979, 4217

8. R.Palmer, Particle Acc.,1980, Vo111,81-9o

9. Peng Huan, Zhuang Jiejia, Scienta Sinica, Vo123, No2,1980,159-171

10. P.B.Wilson, KEK Lectures, KEK Accelerator 79-7,1980

11. V.V.Shevchenko, Contintous transitions in open waveguides, Golden Press, Boulder, Colorado 1971

12. F.M.Samir,J.C.Beal, IEEE Trans.on Micr.Wave.Theor.MTT23, 1975

13. D.Marcuse, Theory of dielectric optical waveguides, Äcad.Press, 74

14. T.Weiland, waves on dielectric plates, TH-Darmstadt 1975, unpub1.

15. T.Weiland, Archiv fuer Elektronik u.Übertragungstechnik, 33, 170-4

16. T.Weiland, Kleinheubacher Berichte 22(1979), 435-443

17. N.Kro11, R.Palmer, private communication, this workshop 